\title{
A Greenhouse Method to Evaluate Sunflower Quantitative Resistance to Basal Stalk Rot Caused by Sclerotinia sclerotiorum
}

\author{
William Underwood, ${ }^{1, \dagger}$ Christopher G. Misar, ${ }^{1}$ Charles Block, ${ }^{2}$ Thomas J. Gulya, ${ }^{3}$ Zahirul Talukder, ${ }^{4}$ Brent S. Hulke, \\ and Samuel G. Markell ${ }^{5}$ \\ ${ }^{1}$ USDA-ARS Sunflower and Plant Biology Research Unit, Edward T. Schafer Agricultural Research Center, Fargo, ND 58102 \\ ${ }^{2}$ Seed Science Center, Iowa State University, Ames, IA 50011 \\ ${ }^{3}$ USDA-ARS Sunflower and Plant Biology Research Unit (retired), Edward T. Schafer Agricultural Research Center, Fargo, ND \\ 58102 \\ ${ }^{4}$ Department of Plant Sciences, North Dakota State University, Fargo, ND 58102 \\ ${ }^{5}$ Department of Plant Pathology, North Dakota State University, Fargo, ND 58102
}

\begin{abstract}
Resistance of sunflower to basal stalk rot (BSR) caused by the fungus Sclerotinia sclerotiorum is quantitative, controlled by multiple genes contributing small effects. Consequently, artificial inoculation procedures allowing sufficient throughput and resolution of resistance are needed to identify highly resistant sunflower germplasm resources and to map loci contributing to resistance. The objective of this study was to develop a greenhouse-based method for evaluating sunflower quantitative resistance to BSR that would be simple, space- and time-efficient, high throughput, high resolution, and correlated with field observations. Experiments were conducted with 5-week-old sunflower plants and Scle-

to assess the correlation of the greenhouse inoculation procedure with field results by using a panel of 32 sunflower genotypes with known field response to BSR previously determined in multiyear, multilocation artificially inoculated trials. Experimental observations indicated that the newly developed greenhouse inoculation procedure provided improved resolution to identify highly resistant genotypes and was strongly correlated with field observations. This method will be useful for screening of sunflower experimental and breeding materials, disease phenotyping of genetic mapping populations, and evaluation of resistance to different pathogen isolates.
\end{abstract} rotinia-infested millet seed as inoculum to assess the impact of pot size and temperature and to determine the most favorable inoculum rate and placement. Subsequently, an additional experiment was performed
Keywords: basal stalk rot, inoculation method, Sclerotinia sclerotiorum, sunflower, white mold
Sclerotinia sclerotiorum is a necrotrophic fungal pathogen capable of causing white mold disease on many plant species, including numerous agriculturally important plants such as sunflower, canola, soybean, and common bean (Boland and Hall 1994; Bolton et al. 2006). Diseases caused by $S$. sclerotiorum are a persistent problem for cultivated sunflower (Helianthus annuus) production in the northern great plains region of the United States, where most U.S. sunflower production is concentrated. Cultivated sunflower is unique among crop species affected by $S$. sclerotiorum in that the pathogen causes three distinct and economically important diseases: head rot, midstalk rot, and basal stalk rot (BSR, also referred to as Sclerotinia wilt; Harveson et al. 2016; Markell et al. 2015). Both head rot and midstalk rot are incited by airborne ascospores that result from carpogenic germination of $S$. sclerotiorum sclerotia to produce apothecia, which release ascospores that land on senescent plant tissues and initiate disease (Harveson et al. 2016; Markell et al. 2015). Head rot occurs when ascospores land on senescent pollen and floral tissues,

${ }^{\dagger}$ Corresponding author: William Underwood;

William.Underwood@usda.gov

Funding: This project was supported by the U.S. Department of Agriculture Agricultural Research Service National Sclerotinia Initiative, CRIS Project no. 3060-21220-031-00D. The mention of trade names or commercial products in this report is solely for the purpose of providing specific information and does not imply recommendation or endorsement by the U.S. Department of Agriculture. The USDA is an equal opportunity provider and employer.

The author(s) declare no conflict of interest.

Accepted for publication 14 July 2020.

This article is in the public domain and not copyrightable. It may be freely reprinted with customary crediting of the source. The American Phytopathological Society, 2021. leading to destruction of the head and loss of seeds under conducive environmental conditions. Midstalk rot occurs when leaf infection progresses down the petiole to the stalk tissue, causing elongated lesions and pith degradation that can result in premature senescence and lodging. In contrast, BSR is a root rot that begins when sclerotia germinate myceliogenically beneath the soil surface and hyphae directly infect sunflower roots (Harveson et al. 2016; Markell et al. 2015). The infection subsequently progresses through the root system to the crown, causing basal stem lesions, whole plant wilting, and death. Root infections and wilting symptoms incited by $S$. sclerotiorum are not observed for other crop hosts of this pathogen. BSR and head rot have been generally regarded as the most economically significant of the three Sclerotinia diseases affecting sunflower production in the United States.

No complete resistance to $S$. sclerotiorum has been discovered in any crop species affected by this pathogen. Resistance is instead quantitative and governed by many genes each contributing small effects (Bert et al. 2002; McCaghey et al. 2017; Soule et al. 2011; Talukder et al. 2016). Consequently, mapping of quantitative trait loci (QTLs) contributing to resistance requires disease assessment that is sufficiently precise to discriminate genotypes exhibiting high levels of resistance or susceptibility from only moderately resistant or susceptible genotypes. Ideally, procedures should allow reasonable throughput, space efficiency, and low cost to facilitate evaluating the large numbers of individuals in structured or natural populations needed for effective mapping of loci contributing to the highly quantitative resistance phenotype. Numerous methods of evaluating resistance of sunflower to BSR have been reported. These include field trials in naturally infested locations or supplemented with inoculum such as S. sclerotiorum sclerotia or mycelium grown on a carrier such as millet or oat seed (Gulya et al. 1989, 2008; Huang and Hoes 1980; Sedun and Brown 1989; Tourvieille de Labrouhe and Vear 1990). Inoculation of basal stem tissues with agar plugs of mycelium has been reported and used for QTL mapping studies (Amoozadeh et al. 2015; Davar et al. 2010). Additionally, greenhouse- or growth chamber-based 
methods including exposure of sunflowers grown in pots to mycelial inoculum have also been reported in the literature (Grezes-Besset et al. 1994; Sedun and Brown 1989). However, none of these methods meet all the criteria previously outlined, with most methods being either insufficiently discriminatory or excessively laborious, time-consuming, or space-inefficient. Additionally, field-based methods may be complicated by disease escape caused by differences in time to flowering or maturity between genotypes.
Given the limitations of available methods for evaluation of sunflower quantitative resistance to BSR, the objective of this study was to develop an improved, greenhouse-based evaluation method to facilitate routine evaluations of structured populations. Major requirements were increased resolution, sufficient throughput, reliability, and space efficiency. This article reports results of three experiments to develop a new method for BSR evaluation and to assess correlation of the method with results from inoculated field
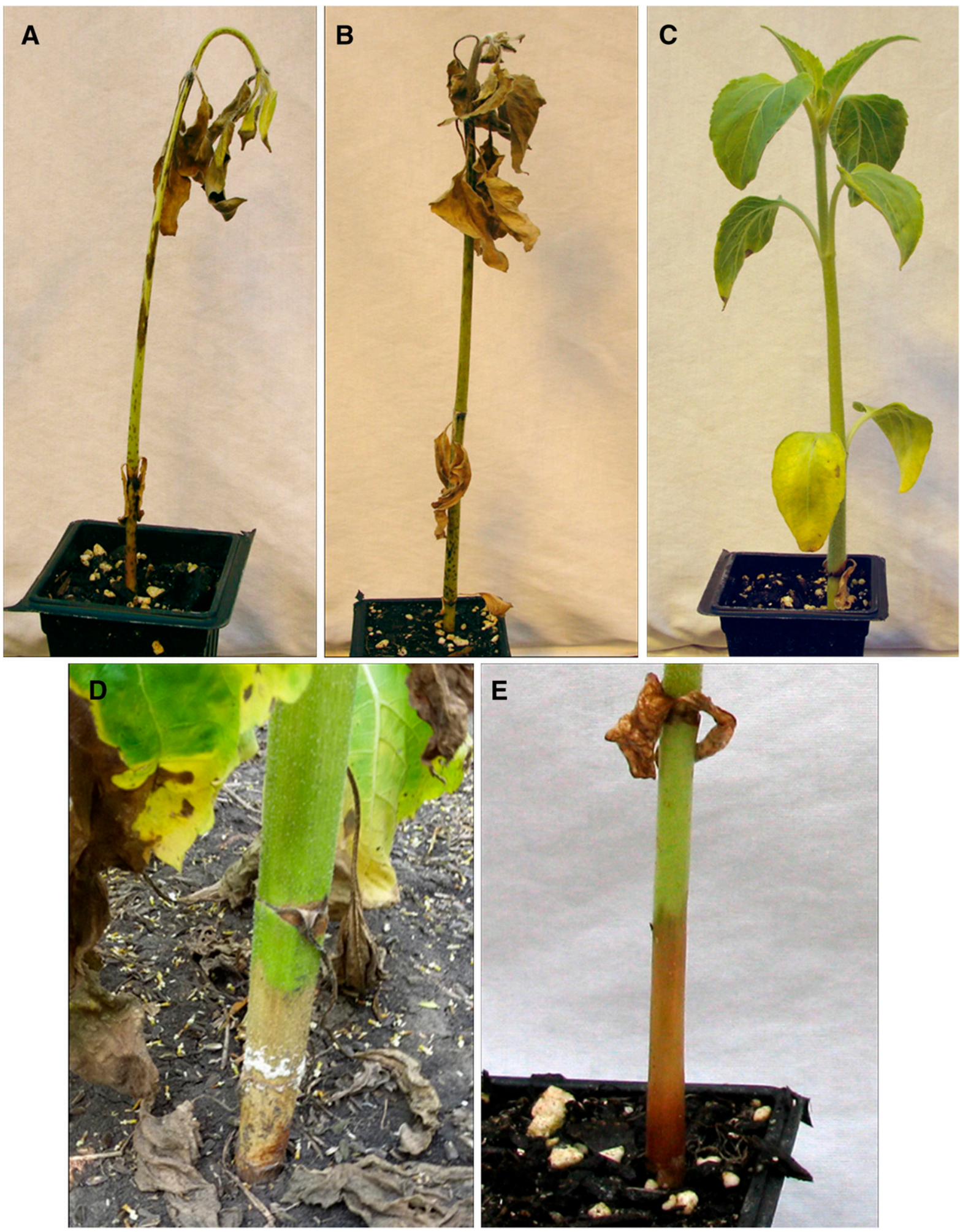

E

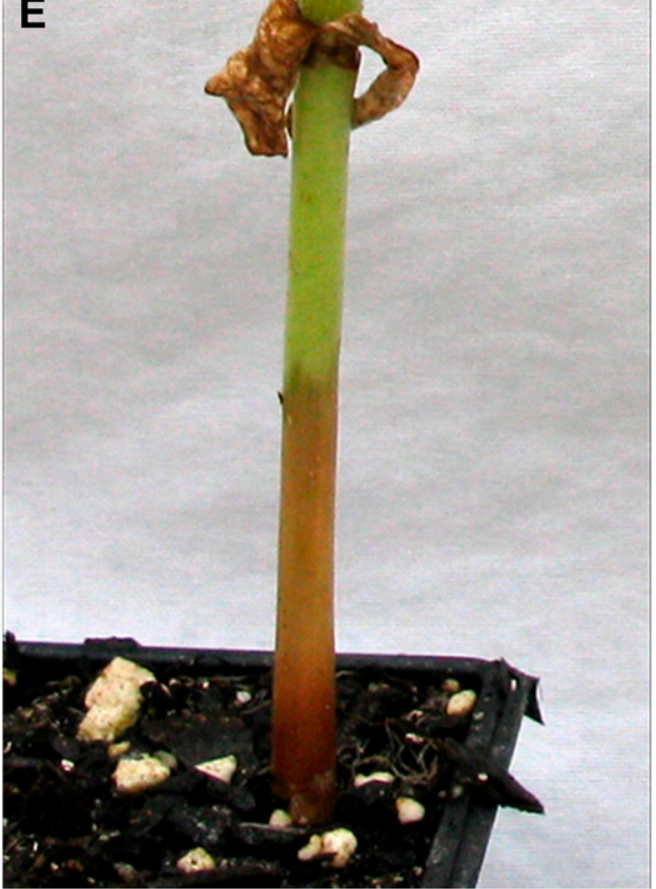

Fig. 1. Symptoms of sunflower basal stalk rot (BSR) observed in the greenhouse and field. A, Susceptible plant exhibiting terminal wilting at 14 days post-inoculation (dpi) in the greenhouse. B, Susceptible plant exhibiting whole plant desiccation at 14 dpi in the greenhouse. C, Resistant plant exhibiting few or no BSR symptoms at 14 dpi in the greenhouse. D, Basal stalk lesion associated with BSR in a field-grown sunflower plant. E, Basal stalk lesion observed on a susceptible plant at 14 dpi in the greenhouse. 
trials. Additionally, the improved resolution of the newly developed evaluation method facilitated the identification of sunflower lines possessing high levels of resistance to BSR that may be useful for improving resistance in breeding programs.

\section{Materials and Methods}

Plant materials and growth conditions. One growth chamber experiment and two independent greenhouse experiments were performed to investigate methods for evaluating resistance of sunflower to BSR. Two initial experiments were conducted to investigate specific parameters for plant growth and inoculation procedures and to evaluate the suitability of kinetic analysis of time to plant death from BSR for separating the response of genotypes. Experiment 1 was conducted in a growth chamber to evaluate the effects of pot size and temperature on BSR response. Experiment 2 was conducted in the greenhouse to investigate effects of rate and placement of S. sclerotiorum inoculum. Finally, Experiment 3 was conducted in the greenhouse with a panel of 32 sunflower accessions with known BSR response in inoculated field trials to assess the correlation of greenhouse results with field observations.

Two commercial sunflower hybrids expected to exhibit different levels of BSR resistance, Cargill 270 (Cargill, Inc.) and BigFoot (Seeds2000, Inc.), were used to investigate effects of pot size and temperature in Experiment 1. Based on previous field observations and preliminary investigations, Cargill 270 was expected to be highly susceptible to BSR, and BigFoot was expected to be moderately resistant.

Experiment 2 involved 10 sunflower inbred lines expected to exhibit a range of BSR response. Inbred lines HA 207, HA 434, HA 89, and RHA 373 were developed by the U.S. Department of Agriculture (USDA) Agricultural Research Service and have previously been observed to exhibit susceptibility to BSR in inoculated field trials

Table 1. Analysis of variance for the effects of sunflower hybrid, temperature, pot size, and interactions between these variables on basal stalk rot time to plant death response

\begin{tabular}{lccc}
\hline Source of variance & $\boldsymbol{d} \boldsymbol{f}$ & $\boldsymbol{F}$ & $\boldsymbol{P}$ \\
\hline Hybrid & 1 & 750.65 & $<0.001$ \\
Temperature & 1 & 89.2 & $<0.001$ \\
Pot size & 1 & 139.18 & $<0.001$ \\
Hybrid $\times$ temperature & 1 & 41.12 & $<0.001$ \\
Hybrid $\times$ pot size & 1 & 19.32 & $<0.001$ \\
Temperature $\times$ pot size & 1 & 0.71 & 0.3991 \\
\hline
\end{tabular}

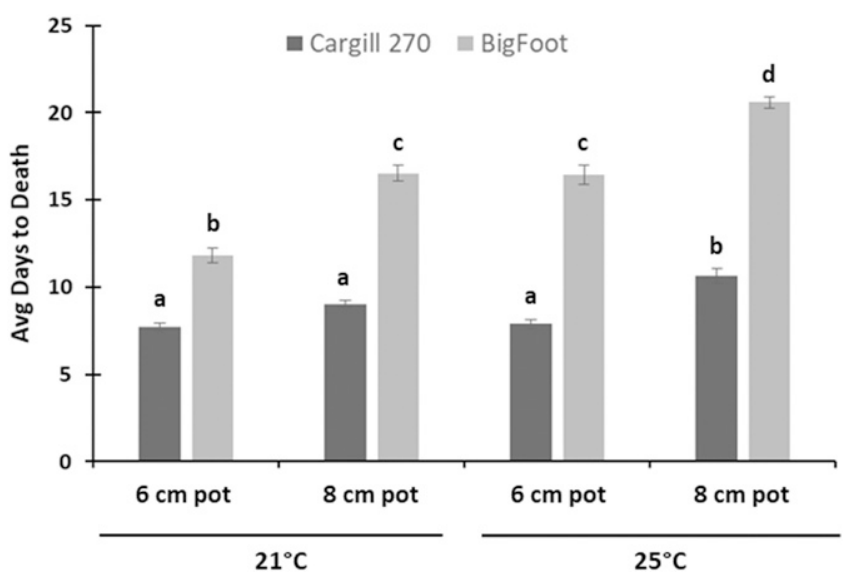

Fig. 2. Effects of pot size and temperature on basal stalk rot (BSR) response. Average days to plant death caused by BSR are shown for the susceptible sunflower hybrid Cargill 270 (dark gray bars) and the moderately resistant hybrid BigFoot (light gray bars) when grown in 6-cm pots or $8-\mathrm{cm}$ pots and at 21 or $25^{\circ} \mathrm{C}$. Error bars indicate SEM. Bars indicated by the same letter are not significantly different according to Tukey's post hoc test $(P<0.05)$.
(Miller 1992; Miller et al. 2004; Seiler et al. 2017; Stafford and Thompson 1983; Talukder et al. 2014, 2016). Inbred lines HA 410, HA 412 HO, HA 441, and RHA 439 were developed by the USDA Agricultural Research Service for improved BSR resistance and exhibit varying degrees of partial resistance in inoculated field trials (Miller and Gulya 1999, 2006; Miller et al. 2006; Talukder et al. 2014). Inbred lines RHA 294 and RHA 801 were developed by the USDA Agricultural Research Service and exhibit partial BSR resistance in field nurseries under conditions of natural infection (Fick and Zimmer 1979; Gulya 1985; Gulya et al. 1989; Roath et al. 1981).

Experiment 3 involved a validation panel of 32 sunflower inbred lines and plant introductions. Thirty sunflower lines evaluated in Experiment 2 were chosen based on results from multisite, multilocation inoculated field trials involving 260 sunflower inbred lines and plant introductions (Talukder et al. 2014). The 10 most resistant and 10 most susceptible lines along with 10 intermediate lines from the previously reported field trials were selected as a validation panel to determine whether this panel of lines would exhibit a similar pattern of BSR response when evaluated via the greenhouse method. Inbred lines RHA 801 and RHA 373 were included in Experiment 3 because they exhibited clear differential responses in Experiment 2. Sunflower plant introduction accessions evaluated in Experiment 3 were acquired from the USDA North Central Regional Plant Introduction Station, Ames, Iowa. Inbred line RHA 377 was developed by the USDA Agricultural Research Service (Miller 1992). For Experiment 1, two pot sizes, two temperatures, and two hybrids were evaluated. Plants were grown in potting mix using either 32-position 6.1 -cm width $\times$ $6.1-\mathrm{cm}$ length $\times 5.66-\mathrm{cm}$ depth sheet pots, hereafter referred to as 6 -cm pots, or 18 -position $7.9-\mathrm{cm}$ width $\times 7.9$-cm length $\times 5.72-\mathrm{cm}$ depth sheet pots, hereafter $8-\mathrm{cm}$ pots, in a walk-in growth chamber at either constant $21^{\circ} \mathrm{C}$ or constant $25^{\circ} \mathrm{C}$ and with a photoperiod of $14 \mathrm{~h}$ (two technical replicates) or $13 \mathrm{~h}$ (four technical replicates). For Experiments 2 and 3, plants were grown in potting mix in 32-position 6.58 -cm width $\times 6.58$-cm length $\times 7.62-\mathrm{cm}$ depth sheet pots in a greenhouse with supplemental lighting to provide a 16-h photoperiod and at a temperature of $22^{\circ} \mathrm{C}$.

Inoculation experiments. For all experiments, S. sclerotioruminfested millet seed was used as inoculum. Mycelial cultures of S. sclerotiorum isolate NEB-274 (provided by M. Boosalis, University of Nebraska; Seiler et al. 2017) were initiated by plating single sterile sclerotia on potato dextrose agar (PDA) in $90-\mathrm{mm}$ diameter Petri dishes. Starter cultures were grown at $21^{\circ} \mathrm{C}$ for 4 days. Agar plugs were then cut from the growing edge of the colony with a 6-mm diameter cork borer and transferred to five new PDA plates. White proso millet seed $(1.45 \mathrm{~kg})$ was placed in an autoclavable pan with 3 liters of distilled water and soaked $24 \mathrm{~h}$ at $21^{\circ} \mathrm{C}$. Water was then drained from the millet seed, $300 \mathrm{ml}$ of distilled water was added, the pan was covered with foil and autoclaved twice for $25 \mathrm{~min}$ on consecutive days. S. sclerotiorum PDA cultures (five plates) were grown at $21^{\circ} \mathrm{C}$ for 4 days, then cut into pieces with a sterile scalpel and stirred into the autoclaved millet under sterile conditions. The pan was then covered with foil, and $S$. sclerotiorum was allowed to colonize the millet seed for 6 days at $21^{\circ} \mathrm{C}$, with stirring under sterile conditions after 3 days. S. sclerotiorum-infested millet, before formation of sclerotia, was subsequently transferred to plastic trays and dried at $35^{\circ} \mathrm{C}$ in a ventilated seed-drying room for 5 days. After drying, millet inoculum was transferred to plastic bags and stored in plastic containers at $4^{\circ} \mathrm{C}$ for $\leq 24$ weeks before use.

In Experiment 1, the effects of two different pot sizes and two temperatures on BSR response were evaluated in a growth chamber. Six technical replicates were conducted for each treatment combination. For each technical replicate, sunflower plants were initially grown to the V4 to V6 growth stage (four to six pairs of leaves, 4 to 5 weeks depending on growth temperature) in 6-cm square sheet pots, with 15 (two technical replicates) or 20 (four technical replicates) plants per hybrid. Plants were inoculated by transplanting the root-bound plant to either the same size $6-\mathrm{cm}$ square pot or a larger $8-\mathrm{cm}$ square pot 
containing a thin layer of perlite in the bottom of the pot and $2.5 \mathrm{~g}$ of S. sclerotiorum-infested millet inoculum. Additional potting mix was added to fill the larger pots. Inoculated plants were arranged in a completely randomized design and evaluated daily for plant death resulting from terminal wilt. Terminal wilt was defined as complete loss of turgor after which the apex of the plant was curved downward (Fig. 1A). Daily evaluations were conducted for 21 days post-inoculation (dpi), and time to plant death was recorded for each individual. For the statistical analysis of time to plant death, plants that remained alive at $21 \mathrm{dpi}$ were assigned a value of 22 .

In Experiment 2, two methods for placement of S. sclerotioruminfested millet inoculum were evaluated: placement of inoculum in the bottom of the pot beneath the root mass or insertion of inoculum into the side of the pot. Additionally, two rates of inoculum were evaluated, a low rate of $0.38 \mathrm{~g}$ or a high rate of $0.76 \mathrm{~g}$ of millet per pot, for a total of four treatments. Fifteen 5-week-old plants per sunflower inbred line were inoculated either by removing the plant and root mass from the pot, placing millet inoculum $(0.38$ or $0.76 \mathrm{~g})$ in the bottom of the pot, and returning the plant to place inoculum in contact with the root system; or by inserting millet into the side of the pot. A quarter- or half-teaspoon measuring spoon was used to transfer millet inoculum. The low rate of $0.38 \mathrm{~g}$ corresponds to one quarter teaspoon, and the high rate of $0.76 \mathrm{~g}$ corresponds to one half teaspoon. Plants were subsequently evaluated daily for $21 \mathrm{dpi}$ for death caused by BSR by assessing terminal wilt as described previously or whole plant desiccation. Whole plant desiccation was defined as complete desiccation of all leaf tissues to the point that the plant was crispy to the touch (Fig. 1B). For the statistical analysis of time to plant death, plants that remained alive at 21 dpi were assigned a value of 22 .

In Experiment 3, 15 5-week-old plants per sunflower accession were inoculated by placing $0.76 \mathrm{~g}$ of $S$. sclerotiorum-infested millet in the bottom of the pot. Inoculated plants were arranged in a randomized complete block design for a total of 15 blocks, and plants were evaluated daily for death caused by BSR as described for Experiment 2. In Experiments 1 and 2, we observed that many plants survived

Table 2. Evaluation of Sclerotinia sclerotiorum millet inoculum rate and placement on 10 sunflower inbred lines

\begin{tabular}{|c|c|c|c|}
\hline \multicolumn{2}{|c|}{ Bottom inoculation, low rate ${ }^{v}$} & \multicolumn{2}{|c|}{ Bottom inoculation, high rate $^{\mathrm{v}}$} \\
\hline Inbred line & Average days to death ${ }^{w, x, y}$ & Inbred line & Average days to death ${ }^{w, x, y}$ \\
\hline RHA 801 & $14.8 \mathrm{a}$ & RHA 801 & $14.2 \mathrm{a}$ \\
\hline RHA 294 & $10.9 \mathrm{ab}$ & RHA 294 & $11.5 \mathrm{ab}$ \\
\hline HA 207 & $9.7 \mathrm{~b}$ & HA 207 & $11.3 \mathrm{ab}$ \\
\hline RHA 439 & $9.4 \mathrm{~b}$ & HA 434 & $10.8 \mathrm{ab}$ \\
\hline HA 434 & $9.3 \mathrm{~b}$ & RHA 439 & $9.6 \mathrm{abc}$ \\
\hline HA 89 & $9.2 \mathrm{~b}$ & HA 441 & 7.9 bc \\
\hline HA 410 & $8.8 \mathrm{bc}$ & HA 410 & $7.9 \mathrm{bc}$ \\
\hline HA $412 \mathrm{HO}$ & $8.6 \mathrm{bc}$ & HA $412 \mathrm{HO}$ & $7.8 \mathrm{bc}$ \\
\hline HA 441 & $8.1 \mathrm{bc}$ & HA 89 & $7.1 \mathrm{c}$ \\
\hline RHA 373 & $6.1 \mathrm{c}$ & RHA 373 & $6.6 \mathrm{c}$ \\
\hline \multicolumn{2}{|c|}{ Side inoculation, low rate } & \multicolumn{2}{|c|}{ Side inoculation, high rate } \\
\hline Inbred line & Average days to death ${ }^{w, x, y}$ & Inbred line & Average days to death ${ }^{w, x, y}$ \\
\hline RHA 294 & $12.1 \mathrm{a}$ & RHA 801 & $11.1 \mathrm{a}$ \\
\hline RHA 801 & 11.9 a & HA 89 & $10.1 \mathrm{ab}$ \\
\hline RHA 439 & $9.6 \mathrm{ab}$ & HA 441 & $9.3 \mathrm{abc}$ \\
\hline HA 89 & $9.5 \mathrm{ab}$ & RHA 294 & $8.5 \mathrm{abc}$ \\
\hline HA 441 & $8.6 \mathrm{abc}$ & HA 410 & $8.3 \mathrm{abcd}$ \\
\hline HA 410 & $8.3 \mathrm{bc}$ & HA 207 & $8.3 \mathrm{abcd}$ \\
\hline HA 207 & $7.1 \mathrm{bc}$ & RHA 439 & $7.8 \mathrm{bcd}$ \\
\hline HA 434 & $6.7 \mathrm{c}$ & HA $412 \mathrm{HO}$ & $7.6 \mathrm{bcd}$ \\
\hline HA $412 \mathrm{HO}$ & $6.7 \mathrm{c}$ & HA 434 & $7.1 \mathrm{~cd}$ \\
\hline RHA 373 & $6.5 \mathrm{c}$ & RHA 373 & $6.0 \mathrm{~d}$ \\
\hline
\end{tabular}

v S. sclerotiorum-infested millet was placed in the bottom of the pot, beneath the sunflower root mass, at a low rate of $0.38 \mathrm{~g}$ or a high rate of $0.76 \mathrm{~g}$.

w A total of 15 plants per line were inoculated, organized in a randomized complete block design, and evaluated daily for basal stalk rot.

${ }^{x}$ Data were analyzed via a generalized linear mixed model in which block was treated as a random effect. Numbers are estimated means for 15 observations per line.

y Means followed by the same letter are not significantly different according to Tukey's post hoc test $(P<0.05)$.

z S. sclerotiorum-infested millet was inserted into the side of the pot at a low rate of $0.38 \mathrm{~g}$ or a high rate of $0.76 \mathrm{~g}$.

Table 3. Comparison of inbred line responses to each combination of inoculation placement and rate ${ }^{\mathrm{z}}$

\begin{tabular}{lcccc}
\hline Inbred line & Bottom inoculation low rate & Bottom inoculation high rate & Side inoculation low rate & Side inoculation high rate \\
\hline RHA 801 & $14.8 \mathrm{a}$ & $14.2 \mathrm{a}$ & $11.9 \mathrm{a}$ & $11.1 \mathrm{a}$ \\
HA 441 & $8.1 \mathrm{a}$ & $7.9 \mathrm{a}$ & $8.6 \mathrm{a}$ & $9.3 \mathrm{a}$ \\
RHA 294 & $10.9 \mathrm{ab}$ & $11.5 \mathrm{ab}$ & $12.1 \mathrm{a}$ & $8.5 \mathrm{~b}$ \\
HA 207 & $9.7 \mathrm{ab}$ & $11.3 \mathrm{a}$ & $7.1 \mathrm{c}$ & $8.3 \mathrm{bc}$ \\
RHA 439 & $9.4 \mathrm{a}$ & $9.6 \mathrm{a}$ & $9.6 \mathrm{a}$ & $7.8 \mathrm{a}$ \\
HA 434 & $9.3 \mathrm{ab}$ & $10.8 \mathrm{a}$ & $6.7 \mathrm{c}$ & $7.1 \mathrm{bc}$ \\
HA 89 & $9.2 \mathrm{ab}$ & $7.1 \mathrm{a}$ & $9.5 \mathrm{ab}$ & $8.1 \mathrm{~b}$ \\
HA 410 & $8.8 \mathrm{a}$ & $7.9 \mathrm{a}$ & $6.3 \mathrm{a}$ & $7.6 \mathrm{a}$ \\
HA 412 HO & $8.6 \mathrm{a}$ & $7.8 \mathrm{a}$ & $6.5 \mathrm{a}$ & $6.0 \mathrm{a}$ \\
RHA 373 & $6.1 \mathrm{a}$ & $6.6 \mathrm{a}$ & \\
\hline
\end{tabular}

${ }^{\mathrm{z}}$ Data for each of 10 inbred lines were analyzed independently across four combinations of inoculation placement and rate via a generalized linear model to determine whether responses were significantly different between treatments. For each inbred line, average days to death responses indicated by the same letter are not significantly different according to Tukey's post hoc test $(P<0.05)$. 
past the 21-day evaluation period. Therefore, we extended the evaluation period in Experiment 3 to provide more information about the response of resistant lines. In this experiment, daily evaluations were conducted for $28 \mathrm{dpi}$, and time to plant death was recorded for each individual. For the statistical analysis of time to plant death, plants that remained alive at 28 dpi were assigned a value of 29 .

Statistical analysis. Time to plant death data for Experiment 1 were analyzed with a generalized linear model implemented in SAS version 9.4 PROC GLM (SAS Institute 2013). Analysis of variance was performed on data from six technical replicates to determine the effects of sunflower hybrid, pot size, and temperature and the interactions between these factors on BSR response. When analysis of variance revealed significant differences between means $(P<$ $0.05)$, Tukey's post hoc test was used to separate means at $P<0.05$ (Tukey 1949). Time to plant death data for Experiments 2 and 3 were analyzed with a generalized linear mixed model implemented in SAS version 9.4 PROC GLIMMIX (SAS Institute 2013). To assess the degree of quantitative BSR resistance, mean days to death for each accession were estimated, and significant differences between accessions were determined. For Experiment 2, means for each inbred line were also compared across the four combinations of inoculum placement, and rate and Pearson correlations were determined for the four inoculation treatments. For Experiment 3, three technical replicates were conducted, and data were combined for statistical analysis after homogeneity of variance was confirmed across technical replicates via Levene's test (Levene 1960). Block was considered as a random effect for Experiment 2, and block and technical replication were considered as random effects for Experiment 3. Least squares means were calculated. and when analysis of variance revealed significant differences between means $(P<0.05)$ Tukey's post hoc test was used to separate means at $P<0.05$. For the purposes of validating the greenhouse evaluation procedure, BSR disease incidence data from inoculated field trials conducted over 2 years at three locations in North Dakota and Minnesota were compared with greenhouse data

\section{Bottom Inoculation Bottom Inoculation}

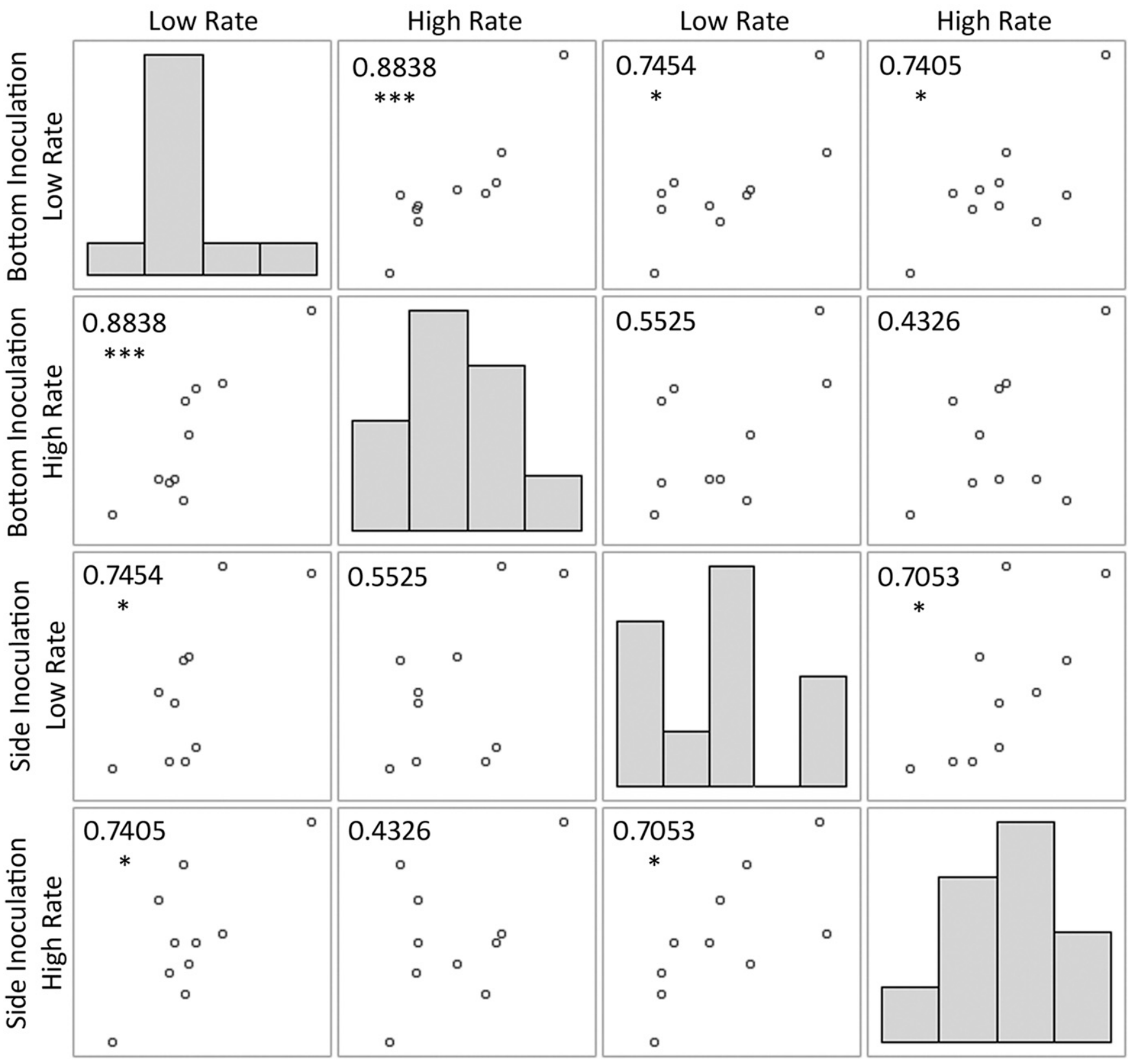

Fig. 3. Correlations between basal stalk rot (BSR) responses for 10 sunflower inbred lines evaluated via four combinations of inoculation method and inoculum rate. Pearson's correlation coefficients for pairwise correlations are indicated in the upper left corner of each scatter plot. Distributions of BSR response of 10 inbred lines for each treatment are shown on the diagonal. *, significant correlation at $P<0.05$; ${ }^{* *}$, significant correlation at $P<0.001$. 
from Experiment 3 via Pearson correlations (Talukder et al. 2014). In these trials, millet inoculum at $80 \mathrm{~g}$ per 6-m row was placed in a furrow (5- to 7-cm depth) approximately $25 \mathrm{~cm}$ from each sunflower row with a granular chemical applicator. Disease was evaluated at approximately 10 weeks after inoculation, and disease incidence was expressed as the percentage of plants exhibiting BSR symptoms of wilting or basal stem lesion.

\section{Results}

In Experiment 1, the effects of pot size and temperature on BSR response of two sunflower hybrids were evaluated in a growth chamber. Plant responses to inoculation included typical BSR symptoms of terminal wilt, leaf necrosis, and whole plant desiccation (Figs. $1 \mathrm{~A}$ and B). Additionally, susceptible plants typically developed basal stem lesions characteristic of BSR and similar to those observed in field-grown sunflowers (Fig. 1D and E). Partial plant wilting was also frequently observed during the experiment. However, plants exhibiting only partial loss of turgor typically recovered and survived for additional days. The effects of sunflower hybrid, pot size, and temperature on BSR response were all significant (Table 1). Additionally, significant interactions were observed for hybrid $\times$ pot size and hybrid $\times$ temperature but not pot size $\times$ temperature. As anticipated, hybrid BigFoot exhibited partial resistance to BSR, whereas Cargill 270 was highly susceptible, and the difference in BSR response between these hybrids was significant for all pot size and temperature treatment combinations (Fig. 2). The effects of temperature and pot size were more pronounced for the moderately resistant hybrid BigFoot. At $25^{\circ} \mathrm{C}$, inoculated plants survived for a longer duration, and more plants survived the duration of the experiment (Fig. 2). Similarly, inoculated plants transplanted to the larger 8 -cm pots also survived for a longer duration (Fig. 2).

Experiment 2 was undertaken to assess the feasibility of evaluating sunflower genotypes for quantitative BSR resistance in the greenhouse and to determine a favorable rate and placement of $S$. sclerotiorum inoculum. Significant differences $(P<0.001)$ in BSR response were observed between the 10 sunflower inbred lines under all four treatment conditions (Table 2). Based on previous field observations, it was expected that inbred line RHA 801 would be among the most resistant lines, whereas RHA 373 would be among the most susceptible. Both lines behaved as anticipated under all four treatment conditions. RHA 373 was the most susceptible genotype under all treatment conditions, with mean time to plant death of approximately 6 dpi (Table 2). In contrast, RHA 801 was the most resistant genotype under three of the four treatment conditions, with a mean time to plant death ranging from approximately 11 to $15 \mathrm{dpi}$ depending on rate and placement of inoculum (Table 2). To compare the different treatment combinations of inoculum placement and rate, we compared the means of time to plant death for each inbred line across the four treatments. Six inbred lines exhibited statistically similar BSR responses across the different treatments, and four lines

Table 4. Comparison of field and greenhouse Sclerotinia basal stalk rot (BSR) response for a 32-accession sunflower validation panel

\begin{tabular}{|c|c|c|c|c|c|}
\hline Accession name & $\mathbf{P I}^{\mathbf{u}}$ & Field disease incidence $(\%)^{\mathrm{v}, \mathrm{w}}$ & Field response & Greenhouse average days to death ${ }^{w, x, y}$ & Greenhouse response \\
\hline HIR 34 & 650613 & $10.8 \mathrm{abc}$ & Resistant & $21.3 \mathrm{a}$ & Highly resistant \\
\hline RHA 801 & 599768 & $3.0 \mathrm{n} / \mathrm{a}^{\mathrm{z}}$ & Resistant & $20.0 \mathrm{a}$ & Highly resistant \\
\hline Krzynowloski Miejscowy & 535890 & $8.3 \mathrm{a}$ & Resistant & $16.9 \mathrm{ab}$ & Highly resistant \\
\hline Slovenska Siva & 531389 & $8.7 \mathrm{ab}$ & Resistant & $14.7 \mathrm{bc}$ & Moderately resistant \\
\hline CO-PB 105 & 600714 & $4.6 \mathrm{a}$ & Resistant & $14.6 \mathrm{bcd}$ & Moderately resistant \\
\hline HA 441 & 639164 & $1.9 \mathrm{a}$ & Resistant & 13.9 bcde & Moderately resistant \\
\hline Gigant 549 & 531352 & 36.3 abcde & Intermediate & 13.7 bcdef & Moderately resistant \\
\hline Harkouski-101 & 650778 & $5.3 \mathrm{a}$ & Resistant & 13.6 bcdefg & Moderately resistant \\
\hline Berzanskij & 650773 & 38.3 abcde & Intermediate & 13.1 bcdefg & Moderately resistant \\
\hline VNIIMK 1696 & 650787 & $13.1 \mathrm{abcd}$ & Resistant & $12.6 \mathrm{cdefg}$ & Intermediate \\
\hline VNIIMK 3497 & 650786 & $7.8 \mathrm{a}$ & Resistant & 12.0 cdefgh & Intermediate \\
\hline S8 V6540 4/7-1 & 650523 & 37.2 abcde & Intermediate & 11.6 cdefghi & Intermediate \\
\hline OLH 2-82 & 535893 & 35.8 abcde & Intermediate & 11.5 cdefghi & Intermediate \\
\hline Saratovskij rannij & 486364 & 38.6 abcde & Intermediate & 11.3 cdefghi & Intermediate \\
\hline Antilco & 650837 & 41.6 abcde & Intermediate & 11.2 defghi & Intermediate \\
\hline ANN-1103 & 650648 & 41.0 abcde & Intermediate & 11.2 defghi & Intermediate \\
\hline Bodroghalmi & 531340 & 36.1 abcde & Intermediate & 10.8 efghi & Intermediate \\
\hline High Oil 74 & 650562 & $14.2 \mathrm{abcd}$ & Resistant & 10.6 efghi & Intermediate \\
\hline RHA 377 & 560145 & $11.1 \mathrm{abcd}$ & Resistant & 10.6 fghij & Intermediate \\
\hline S37-388 & 650513 & 39.3 abcde & Intermediate & 10.5 fghij & Intermediate \\
\hline Wielkopolski & 535894 & 38.7 abcde & Intermediate & 10.4 ghij & Intermediate \\
\hline RHA 373 & 560141 & 28.6 abcde & Intermediate & 9.5 hijk & Susceptible \\
\hline CO-PB 39 & 650675 & $79.5 \mathrm{cde}$ & Susceptible & 9.4 hijk & Susceptible \\
\hline Portugal & 531385 & $87.7 \mathrm{e}$ & Susceptible & 9.4 hijk & Susceptible \\
\hline CO-PB 104 & 650710 & $90.9 \mathrm{e}$ & Susceptible & $9.1 \mathrm{ijk}$ & Susceptible \\
\hline CO-PB 93 & 650705 & $81.7 \mathrm{de}$ & Susceptible & $9.0 \mathrm{ijk}$ & Susceptible \\
\hline CO-PB 67 & 600704 & $87.7 \mathrm{e}$ & Susceptible & $8.3 \mathrm{jk}$ & Susceptible \\
\hline CO-PB 110 & 650713 & $87.0 \mathrm{e}$ & Susceptible & $8.2 \mathrm{jkl}$ & Highly susceptible \\
\hline CO-PB 82 & 600709 & $86.5 \mathrm{e}$ & Susceptible & $8.0 \mathrm{jkl}$ & Highly susceptible \\
\hline CO-PB 90 & 650703 & 76.0 bcde & Susceptible & $7.7 \mathrm{kl}$ & Highly susceptible \\
\hline Hopi dye & 432508 & $88.3 \mathrm{e}$ & Susceptible & $7.4 \mathrm{kl}$ & Highly susceptible \\
\hline Cabure 1004 & 650798 & $93.3 \mathrm{e}$ & Susceptible & 6.41 & Highly susceptible \\
\hline
\end{tabular}

u PI = Plant introduction.

$\checkmark$ Disease incidence values from multilocation, multiyear field trials have been previously reported in Talukder et al. (2014). Trials were conducted with a setswithin-replicates experimental design. Data were analyzed via a generalized linear mixed model in which set, replicate, and environment were treated as random effects. Numbers are estimated means from combined analysis of 4 trials conducted at 3 locations over 2 years.

${ }^{w}$ Means within a column followed by the same letter are not significantly different according to Tukey's post hoc test $(P<0.05)$.

x A total of 15 plants per line were inoculated per technical replicate, organized in a randomized complete block design, and evaluated daily for BSR. The experiment was repeated a total of 3 times.

y Data were analyzed via a generalized linear mixed model in which block and technical replicate were treated as random effects. Numbers are estimated means from combined analysis of 3 technical replicates with 15 observations for each line per technical replicate.

z Sunflower inbred line RHA 801 was not included in field trials with the other 31 accessions. Field incidence data for this line are from an independent field trial. 
exhibited significantly different responses, indicating that at least some lines responded somewhat differently depending on inoculum placement or rate (Table 3). We also evaluated correlations between BSR responses of the 10 lines among the four treatments. BSR responses to bottom inoculation at the high and low inoculum rates were highly correlated $(P<0.001)$, whereas responses to side inoculation were only moderately correlated $(P<0.05$; Fig. 3$)$. We chose the bottom inoculation method at the high rate of $0.76 \mathrm{~g}$ of millet inoculum for further evaluation for several reasons. First, bottom inoculation produced a broader range of BSR response among the 10 inbred lines than side inoculation. Second, bottom inoculation was more rapid and simple than placement of millet into the side of the pot and could more easily be scaled to large populations. Finally, based on field data and preliminary observations, we expected inbred line HA 89 to be among the more susceptible lines. Bottom inoculation with $0.76 \mathrm{~g}$ of inoculum placed HA 89 among the most susceptible genotypes, consistent with our expectations.

In Experiment 3, significant differences $(P<0.001)$ in levels of BSR resistance, as measured by mean time to plant death, were observed among the 32 sunflower lines. Time to plant death ranged from approximately $6 \mathrm{dpi}$ for the most susceptible line, Cabure 1004 (PI 650798), to >21 dpi for the most resistant line, HIR 34 (PI 650613; Table 4). Kinetic evaluation of rates of plant mortality for the 32 lines showed that the greenhouse method grouped lines largely in accordance with the previous field observations (Fig. 4). Lines that exhibited high disease incidence in field trials rapidly died from BSR in the greenhouse evaluation, whereas lines with lower levels of field disease incidence survived for a longer period in the greenhouse evaluation. The results of the greenhouse evaluation of mean days to plant death for each line were strongly and significantly $(P<0.001)$ correlated with BSR disease incidence observed in the field trials, with a Pearson's correlation coefficient of -0.765 (Fig. 5). Field disease incidence and greenhouse days to plant death are negatively correlated because more resistant lines exhibit a low incidence in field trials but a longer time to plant death in the greenhouse evaluation. Importantly, although the greenhouse method was largely consistent with results from inoculated field trials, the greenhouse evaluation exhibited higher resolution by forming more statistical groups and could more clearly separate highly resistant from only moderately resistant or average lines (Table 4). For the purpose of delineating useful groups of responses to BSR, members of the most resistant statistical group were defined as highly resistant, lines in the second-tier statistical group as moderately resistant, lines in the most susceptible statistical group as highly susceptible, lines in the second most susceptible statistical group as susceptible, and all other lines as intermediate (Table 4).

Given that evaluating large numbers of plants daily for death caused by BSR is time consuming and onerous, an assessment was undertaken to determine the consequences of evaluating inoculated plants every other day or every third day. For this purpose, data from Experiment 3 were adjusted to simulate evaluation every other day, beginning at $4 \mathrm{dpi}$, for a total of 13 evaluations or every third day for a total of nine evaluations. Adjusted data were then subjected to the same statistical treatment as the original data derived from daily evaluations. Reducing the number and frequency of evaluations had only minor effects on rank order of genotypes and statistical power to separate genotypes (Table 5).

\section{Discussion}

A new, greenhouse-based method to evaluate sunflowers for quantitative resistance to BSR caused by the necrotrophic fungus $S$. sclerotiorum was successfully developed. This method involves inoculation

Resistant ----- Intermediate $-\quad$ Susceptible

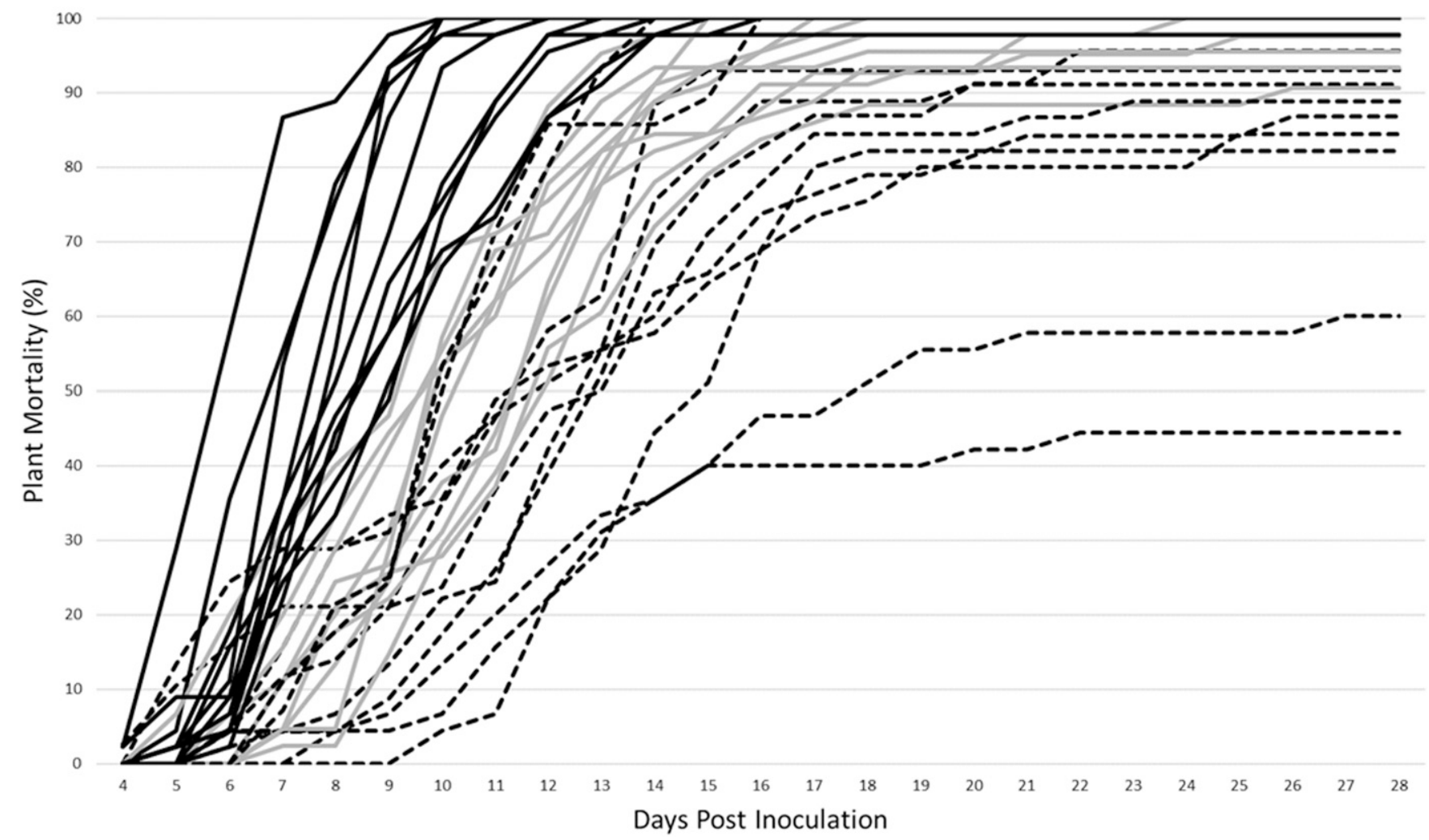

Fig. 4. Kinetic analysis of basal stalk rot (BSR) disease onset after inoculation in the greenhouse for 32 sunflower accessions with known field response. The percentages of plants exhibiting death caused by BSR are plotted on the $x$-axis, and days post-inoculation are plotted on the $y$-axis. Accessions exhibiting a susceptible response in the field are indicated by solid black lines, accessions exhibiting an intermediate response in the field are indicated by gray lines, and accessions exhibiting a resistant response in the field are indicated by dashed black lines. Results shown are combined data from three technical replicates ( $n=45$ plants per accession). In each technical replicate, 15 plants per accession were inoculated and evaluated daily for death caused by BSR for 28 days. 
of sunflower plants grown in small pots in the greenhouse with S. sclerotiorum mycelia grown on autoclaved millet seed as a carrier followed by evaluation of time to plant death from BSR. The inoculation procedure is simple and rapid: Root-bound sunflower plants are removed from the pot to allow a fixed amount of Sclerotinia-infested millet seed to be placed in the bottom of the pot before the plants are returned to the pot, so the root mass is placed in direct contact with the mycelial inoculum. The results of this study demonstrated that the procedure provides good resolution to separate quantitative BSR responses of sunflower genotypes into numerous statistical groups, facilitating the identification of highly resistant lines. Additionally, BSR responses of sunflower genotypes determined via the greenhouse method were highly correlated with observations from multiyear, multilocation inoculated field trials, indicating that the method can identify genotypes that will exhibit useful resistance in field conditions. The new method provides a valuable tool to facilitate improvement of sunflower BSR resistance.

A key objective was to develop a method suitable for routine evaluation of structured populations for genetic mapping and molecular marker development, such as populations of several hundred recombinant inbred lines needed for successful mapping of loci controlling quantitative traits such as BSR resistance. Other methods have been developed for both field- and greenhouse-based phenotyping of this disease. Field-based methods include relying on natural infection by establishing nurseries in fields with known disease history and incorporating sclerotia into field plots to provide inoculum (Gulya et al. 1989; Huang and Hoes 1980). However, these methods often fail to produce adequate disease pressure, and an artificial inoculation method that uses Sclerotinia-infested millet inoculum deposited into furrows next to sunflower rows in field nurseries was developed to improve reliability (Gulya et al. 2008). This method was used to evaluate a set of 260 sunflower plant introduction and inbred lines and to phenotype a recombinant inbred line population to facilitate QTL mapping (Talukder et al. 2014; Talukder et al. 2016). However, even this improved method often fails to produce sufficient disease pressure and offers only limited resolution, prompting Talukder et al. (2014) to note that although "the level of disease allowed for successful differentiation of many genotypes, future breeding experiments may require a procedure that produces more disease (i.e., a 'hotter' test) or one that has greater statistical power through the reduction of experimental error."

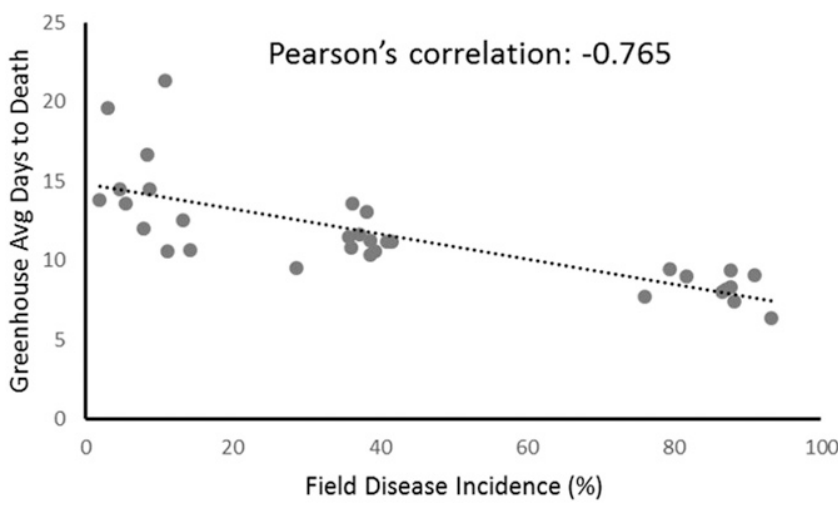

Fig. 5. Correlation between field and greenhouse basal stalk rot (BSR) response for 32 sunflower accessions. Average days to plant death caused by BSR for each accession are plotted on the $y$-axis, and percentages of disease incidence in field trials for each accession are plotted on the $x$-axis. Greenhouse data are combined estimated means ( $n=45$ plants per accession) from three technical replicates evaluated via a generalized linear mixed model. In each technical replicate, 15 plants per accession were inoculated and evaluated daily for death caused by BSR for 28 days. Field disease incidence is reported as the percentage of inoculated plants and represents combined means from 4 total field trials at 3 locations over 2 years. Field incidence data have been reported previously in Talukder et al. (2014). Pearson's correlation coefficient is indicated and is highly significant $(P<0.001)$. Note that a negative correlation is observed between disease incidence (lower for resistant accessions) and average days to death (higher for resistant accessions).
The results of this study indicate that the newly developed greenhouse method provides better differentiation of genotypes compared with furrow inoculation in field nurseries. Additionally, the greenhouse method produces results in a short time period, reduces the need for multisite and multilocation trials that have typically been conducted, requires production of less inoculum than field trials, and allows easier incorporation of multiple pathogen isolates. The method is space efficient and can be scaled to large population sizes. In a $60.4-\mathrm{m}^{2}$ greenhouse room with $25 \mathrm{~m}^{2}$ of bench space, we were able to fit 200 trays of 32 plants each, for a total of 6,400 plants that could be evaluated in a single experiment. Finally, the new method ensures direct inoculation of root tissues to minimize disease escape or avoidance that can complicate field-based evaluations. Another method for sunflower BSR evaluation that has been reported for QTL mapping involved affixing agar plugs carrying S. sclerotiorum mycelium to basal stem tissues with semitransparent film and subsequently assessing lesion formation on stems (Amoozadeh et al. 2015; Davar et al. 2010). One potential point of concern for this approach is the fact that BSR begins as a root infection, and the agar plug method places inoculum against the base of the sunflower stem. Evidence

Table 5. Comparison of sunflower Sclerotinia basal stalk rot (BSR) disease evaluations every other day or every third day

\begin{tabular}{|c|c|c|c|}
\hline \multirow[b]{2}{*}{ Accession name } & \multirow[b]{2}{*}{$\mathbf{P I}^{\mathbf{u}}$} & \multirow{2}{*}{ 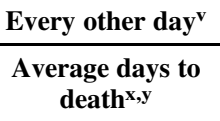 } & \multirow{2}{*}{$\frac{\text { Every third day }^{\mathrm{w}}}{\mathbf{A} \begin{array}{c}\text { Average days to } \\
\text { death }^{\mathrm{x}, \mathrm{y}}\end{array}}$} \\
\hline & & & \\
\hline HIR 34 & 650613 & $22.1 \mathrm{a}$ & $22.3 \mathrm{a}$ \\
\hline RHA 801 & 599768 & $20.2 \mathrm{ab}$ & $20.4 \mathrm{a}$ \\
\hline $\begin{array}{l}\text { Krzynowloski } \\
\text { Miejscowy }\end{array}$ & 535890 & $17.1 \mathrm{bc}$ & $17.7 \mathrm{ab}$ \\
\hline Slovenska Siva & 531389 & $15.1 \mathrm{~cd}$ & $15.3 \mathrm{bcd}$ \\
\hline CO-PB 105 & 600714 & 14.9 cde & $15.6 \mathrm{bc}^{\mathrm{z}}$ \\
\hline HA 441 & 639164 & $14.2 \mathrm{cdef}$ & 14.8 bcde \\
\hline Gigant 549 & 531352 & $14.1 \mathrm{cdef}$ & $14.9 \mathrm{bcde}^{\mathrm{z}}$ \\
\hline Harkouski-101 & 650778 & $14.0 \mathrm{cdefg}$ & 14.7 bcef \\
\hline Berzanskij & 650773 & 13.6 cdefg & 13.8 bcdef \\
\hline VNIIMK 1696 & 650787 & 12.9 defgh & $13.7 \mathrm{cdef}$ \\
\hline VNIIMK 3497 & 650786 & 12.5 defghi & 13.1 cdefg \\
\hline S8 V6540 4/7-1 & 650523 & 12.1 defghij & 12.5 cdefgh \\
\hline OLH 2-82 & 535893 & 12.0 defghij & 12.4 cdefgh \\
\hline Saratovskij rannij & 486364 & 11.7 efghij & $12.1 \mathrm{defgh}$ \\
\hline Antilco & 650837 & 11.5 efghij & 12.1 cdefgh \\
\hline ANN-1103 & 650648 & 11.6 efghij $^{\mathrm{z}}$ & 12.1 cdefgh \\
\hline Bodroghalmi & 531340 & 11.2 fghijk & 11.6 efghi \\
\hline High Oil 74 & 650562 & 11.0 fghijkl & 11.5 efghi \\
\hline RHA 377 & 560145 & 11.0 fghijk & 11.3 fghij \\
\hline S37-388 & 650513 & 11.1 fghijk $^{\mathrm{z}}$ & 11.3 fghij \\
\hline Wielkopolski & 535894 & 10.7 ghijkl & 11.1 fghij \\
\hline RHA 373 & 560141 & 10.1 hijkl & 10.4 ghijk \\
\hline CO-PB 39 & 650675 & 9.9 ijklm & 10.3 ghijk \\
\hline Portugal & 531385 & $9.8 \mathrm{ijklm}$ & 10.3 ghijk \\
\hline CO-PB 104 & 650710 & $9.5 \mathrm{jklm}$ & 10.0 hijk \\
\hline CO-PB 93 & 650705 & $9.6 \mathrm{klmn}{ }^{z}$ & 9.9 hijk \\
\hline CO-PB 67 & 600704 & $8.7 \mathrm{klmn}$ & $9.1 \mathrm{ijkl}$ \\
\hline CO-PB 110 & 650713 & $8.8 \mathrm{klmn}^{\mathrm{z}}$ & $9.2 \mathrm{ijkl}^{\mathrm{z}}$ \\
\hline CO-PB 82 & 600709 & $8.5 \mathrm{lmn}$ & $8.9 \mathrm{jkl}$ \\
\hline CO-PB 90 & 650703 & $8.3 \mathrm{lmn}$ & $8.5 \mathrm{kl}$ \\
\hline Hopi dye & 432508 & $7.8 \mathrm{mn}$ & $8.4 \mathrm{kl}$ \\
\hline Cabure 1004 & 650798 & $7.0 \mathrm{n}$ & 7.31 \\
\hline
\end{tabular}

u $\mathrm{PI}=$ Plant introduction.

$\checkmark$ Data were adjusted to simulate evaluation of plants every other day for BSR.

w Data were adjusted to simulate evaluation of plants every third day for BSR

x Data were analyzed via a generalized linear mixed model in which block and technical replicate were treated as random effects. Numbers are estimated means from combined analysis of 3 technical replicates with $15 \mathrm{ob}-$ servations for each line per technical replicate.

y Means within a column followed by the same letter are not significantly different according to Tukey's post hoc test $(P<0.05)$.

z Rank order differs compared with daily evaluation of plants. 
suggests that sunflower resistance to $S$. sclerotiorum is likely to be tissue specific, because no correlation or a negative correlation has been observed for head rot and BSR resistance (Masirevic and Gulya 1992; Talukder et al. 2014). Consequently, it is unclear whether resistance to stem lesioning accurately predicts BSR resistance in roots. Additionally, manipulation of agar plugs and wrapping stems with semitransparent film are cumbersome procedures. In comparison, the newly developed method directly inoculates the target root tissue and uses an easy-to-handle, millet-based inoculum that can also be stored for months before use, unlike actively growing plate cultures. A greenhouse-based method with some similarities to the newly developed procedure has been reported (Grezes-Besset et al. 1994). In this method, sunflowers grown in pots are transferred to trays containing S. sclerotiorum-infested oat and covered with a mixture of perlite and a synthetic, water-retaining polymer. However, the authors noted that careful irrigation was needed to simultaneously maintain plant growth and avoid excessive disease pressure (Grezes-Besset et al. 1994). Additionally, the reported procedure tested 3-week-old sunflower plants, which, under our growing conditions, are typically not fully root-bound in small pots and are consequently difficult to handle upon removal from pots. Finally, the need to cover transferred plants with the perlite and water-retaining polymer mixture adds material costs and handling steps compared with the simplified procedure reported here.

Potential limitations and shortcomings of this method are worth noting. First, evaluation under controlled conditions will not capture genotype-by-environment interactions influencing disease resistance. Talukder et al. (2014) reported that the most resistant sunflower genotypes in field trials involving 260 accessions exhibited stable resistance across environments, suggesting that genotype-byenvironment interactions may not be of major importance for resistance to this disease. Another important consideration is that the data analysis described here, though successful at identifying and discriminating resistant genotypes, underrepresents the level of resistance for genotypes with large numbers of individuals that survive to the end of the evaluation period. Thus, if such lines are identified, or if breeding progress improves resistance, alternative data analyses may be needed to discriminate genotypes with higher levels of resistance. In this instance, a modification of area under the disease progress curve may be useful to discriminate highly resistant or near immune genotypes. Finally, our results demonstrate that this method is sensitive to temperature and indicate that disease pressure is reduced and probably insufficient at temperatures $\geq 25^{\circ} \mathrm{C}$, which are commonly reached or exceeded in the greenhouse during the summer. Reduced disease pressure at elevated temperatures results in loss of resolution, and therefore it is recommended that researchers avoid evaluating materials at times when greenhouse temperatures regularly exceed $25^{\circ} \mathrm{C}$. In summary, a reliable and efficient new greenhouse-based method for evaluating sunflower quantitative resistance to BSR caused by S. sclerotiorum was successfully developed and validated. The new method should facilitate future genetic mapping and breeding efforts to improve resistance to this disease.

\section{Acknowledgments}

The authors thank Reid Lakin and Jennifer Arnold for technical assistance.

\section{Literature Cited}

Amoozadeh, M., Darvishzadeh, R., Davar, R., Abdollahi Mandoulakani, B., Haddadi, P., and Basirnia, A. 2015. Quantitative trait loci associated with isolate specific and isolate non-specific partial resistance to Sclerotinia sclerotiorum in sunflower. J. Agr. Sci. Tech. 17:213-226.

Bert, P. F., Jouan, I., Tourvieille de Labrouhe, D., Serre, F., Nicolas, P., and Vear, F. 2002. Comparative genetic analysis of quantitative traits in sunflower (Helianthus annuus L.) 1. QTL involved in resistance to Sclerotinia sclerotiorum and Diaporthe helianthi. Theor. Appl. Genet. 105:985-993.

Boland, G. J., and Hall, R. 1994. Index of plant hosts of Sclerotinia sclerotiorum. Can. J. Plant Pathol. 16:93-108.
Bolton, M. D., Thomma, B. P. H., and Nelson, B. D. 2006. Sclerotinia sclerotiorum (Lib.) de Bary: biology and molecular traits of a cosmopolitan pathogen. Mol. Plant Pathol. 7:1-16.

Davar, R., Darvishzadeh, R., Majd, A., Ghosta, Y., and Sarrafi, A. 2010. QTL mapping of partial resistance to basal stem rot in sunflower using recombinant inbred lines. Phytopathol. Mediterr. 49:330-341.

Fick, G. N., and Zimmer, D. E. 1979. Registration of six nonoilseed sunflower parental lines. Crop Sci. 19:422.

Grezes-Besset, B., Tournade, G., Arnauld, O., Urs, R., George, P., Castellanet, P., and Toppan, A. 1994. A greenhouse method to assess sunflower resistance to Sclerotinia root and basal stem infections. Plant Breed. 112:215-222.

Gulya, T. J. 1985. Evaluation of sunflower germplasm for resistance to Sclerotinia stalk rot and race 3 downy mildew. Pages 349-353 in: Proceedings of the 11th International Sunflower Conference. The International Sunflower Association, Paris, France.

Gulya, T. J., Radi, S., and Balbyshev, N. 2008. Large scale field evaluations for Sclerotinia stalk rot resistance in cultivated sunflower. Pages 175-179 in: Proceedings of the 17th International Sunflower Conference. L. Velasco, ed. The International Sunflower Association, Paris, France.

Gulya, T. J., Vick, B. A., and Nelson, B. D. 1989. Sclerotinia head rot of sunflower in North Dakota: 1986 incidence, effect on yield and oil components, and sources of resistance. Plant Dis. 73:504-507.

Harveson, R. M., Markell, S. G., Block, C. C., and Gulya, T. J. 2016. Compendium of Sunflower Diseases and Pests. APS Press, St. Paul, MN.

Huang, H. C., and Hoes, J. A. 1980. Importance of plant spacing and sclerotial position to development of Sclerotinia wilt of sunflower. Plant Dis. 64:81-84.

Levene, H. 1960. Robust tests for equality of variances. Pages 178-292 in: Contributions to Probability and Statistics: Essays in Honor of Harold Hotelling. I., Olkin andH. Hotelling, eds. Stanford University Press, Stanford, CA.

Markell, S. G., Harveson, R. M., Block, C. C., and Gulya, T. J. 2015. Sunflower diseases. Pages 93-128 in: Sunflower: Chemistry, Production, Processing and Utilization. E. Martínez-Force, N. T. Dunflord and J. J. Salas, eds. AOCS Press, Urbana, IL.

Masirevic, S., and Gulya, T. J. 1992. Sclerotinia and Phomopsis: two devastating sunflower pathogens. Field Crops Res. 30:271-300.

McCaghey, M., Willbur, J., Ranjan, A., Grau, C. R., Chapman, S., Diers, B., Groves, C., Kabbage, M., and Smith, D. L. 2017. Development and evaluation of Glycine max germplasm lines with quantitative resistance to Sclerotinia sclerotiorum. Front. Plant Sci. 8:1495.

Miller, J. F. 1992. Registration of five oilseed sunflower germplasm restorer lines (RHA 373 to 377) and two nuclear male-sterile populations (NMS 274 and 801). Crop Sci. 32:1298.

Miller, J. F., and Gulya, T. J. 1999. Registration of eight Sclerotinia-tolerant germplasm lines. Crop Sci. 39:301-302.

Miller, J. F., and Gulya, T. J. 2006. Registration of two restorer (RHA 439 and RHA 440) and one maintainer (HA 441) Sclerotinia tolerant oilseed sunflower germplasms. Crop Sci. 46:482-483.

Miller, J. F., Gulya, T. J., and Vick, B. A. 2004. Registration of two maintainer (HA 434 and HA 435) and three restorer (RHA 436 to RHA 438) high oleic oilseed sunflower germplasms. Crop Sci. 44:1034-1035.

Miller, J. F., Gulya, T. J., and Vick, B. A. 2006. Registration of three maintainer (HA 456, HA 457, and HA 412HO) high-oleic oilseed sunflower germplasms. Crop Sci. $46: 2728$.

Roath, W. W., Miller, J. F., and Gulya, T. J. 1981. Registration of RHA 801 sunflower germplasm. Crop Sci. 21:479.

SAS Institute. 2013. The SAS System for Windows. Release 9.4. SAS Institute, Cary, NC.

Sedun, F. S., and Brown, J. F. 1989. Comparison of three methods to assess resistance in sunflower to basal stem rot caused by Sclerotinia sclerotiorum and S. minor. Plant Dis. 73:52-55.

Seiler, G. J., Misar, C. G., Gulya, T. J., Underwood, W. R., Flett, B. C., Gilley, M. A., and Markell, S. G. 2017. Identification of novel sources of resistance to Sclerotinia basal stalk rot in South African sunflower germplasm. Plant Health Prog. 18:87-90.

Soule, M., Porter, L., Medina, J., Santana, G. P., Blair, M. W., and Miklas, P. N. 2011. Comparative QTL map for white mold resistance in common bean, and characterization of partial resistance in dry bean lines VA19 and I9365-3. Crop Sci. 51:123-139.

Stafford, R. E., and Thompson, T. E. 1983. Registration of sunflower parental line HA 207. Crop Sci. 23:195.

Talukder, Z. I., Hulke, B. S., Marek, L. F., and Gulya, T. J. 2014. Sources of resistance to sunflower diseases in a global collection of domesticated USDA plant introductions. Crop Sci. 54:694-705.

Talukder, Z. I., Seiler, G. J., Song, Q., Ma, G., and Qi, L. 2016. SNP discovery and QTL mapping of Sclerotinia basal stalk rot in sunflower using genotyping-bysequencing. Plant Genome 9:1-16.

Tourvieille de Labrouhe, D., and Vear, F. 1990. Heredity of resistance to Sclerotinia sclerotiorum in sunflowers. III. Study of reactions to artificial infections of roots and cotyledons. Agronomie 10:323-330.

Tukey, J. 1949. Comparing individual means in the analysis of variance. Biometrics 5:99-114. 